\title{
High-Speed Permanent Magnet Synchronous Motor Iron Loss Calculation Method Considering Multi-Physics Factors
}

\author{
Guangwei Liu, Member, IEEE, Meiyang Liu, Yue Zhang, Student Member, IEEE, Huijun \\ Wang, Member, IEEE, Chris Gerada , Member, IEEE
}

\begin{abstract}
For the high-speed permanent magnet synchronous motors (HSPMSMs), their magnetic fields are more complicated with the rotating magnetization and harmonics considered. Furthermore, there are interaction effects on motor iron loss from high frequency, temperature and compressive stress. In order to obtain the accurate HSPMSM iron loss calculation model, different iron loss models are proposed considering these physical factors, and the iron loss model considering the interaction effect of the multi-physics factors is given. The magnetic field, temperature field and stress field are analyzed for HSPMSM by FEM. Then the proposed models are employed to calculate the iron loss of the silicon steel sheet and the prototype. The accuracy of the proposed iron loss model, which can consider the interaction effect of the multi-physics factors, is verified by the experimental test measurements on the prototype.
\end{abstract}

Index Terms-High-speed motor, iron loss calculation, multi-physics factors, permanent magnet motor

\section{INTRODUCTION}

$\mathrm{H}$ IGH-SPEED permanent magnet synchronous motors (HSPMSMs) are increasingly popular owing to their excellent performance including high power density, compact size, high efficiency and the suitability for high-speed direct-drive applications without gearboxes, which are widely

Manuscript received Feb. 2, 2019; revised Jun. 3, 2019; accepted Jul. 16, 2019. This work is supported by National Natural Science Foundation of China (51537007, 51207094,51777004), and PhD. Start-up Fund of Liaoning Province (201601153). (All authors have made technical contribution to this work) (Corresponding author: Guangwei Liu, Zhang Yue)

G. Liu is with the School of Electrical Engineering, Shenyang University of Technology, Shenyang 110870, China (e-mail: liu-gw@outlook.com).

M. Liu are with Zhuhai Gree Electric Appliance Co., Ltd., Zhuhai 519070, China (e-mail: 498081600@qq.com).

Y. Zhang is with the Power Electronics, Machines and Control Group, The University of Nottingham, Nottingham NG7 2RD, U.K. (Yue.Zhang@nottingham.ac.uk)

$\mathrm{H}$. Wang is with the School of Instrumentation Science and Opto-electronics Engineering, Beihang University, Beijing 100191, China. (e-mail: huijun024@gmail.com)

C. Gerada, is with the Power Electronics, Machines and Control Group, The University of Nottingham, Nottingham NG7 2RD, U.K. (chris.gerada@ nottingham.ac.uk) utilized in the industrial field, such as gas compressors, distributed power generation, electrical turbocharging, turbines and flywheel energy storage system [1-3]. However, there are some characteristic issues for HSPMSMs. High power density leads to high power loss density [4]. Due to the high frequency of the magnetic field alternating in the steel core lamination [5], the high temperature rise in total machine, and the stress of other parts in motor acting on the core, iron loss can be significantly higher than that in ordinary motor [6]. Therefore, the accurate iron loss calculation poses a particular challenge for HSPMSM.

The iron loss in the silicon steel sheet is generated by the alternative magnetic field, and it is affected by the magnetization of the magnetic field. It is learnt from the literatures that both alternating magnetization and rotating magnetization exist simultaneously in the motor iron core, wherein the rotating one is the dominant one [7]. The iron loss taking into account of the rotating magnetization is closer to the measured one [8]. The magnetic field harmonic content of high-speed machines was analyzed by Fourier decomposition method, whose effect can not be ignored [9], [10]. Iron loss increases exponentially with frequency [11]. In addition, high frequency also causes the skin effect on the surface of the silicon steel sheet, which further increases the iron loss [12]. Thus, the influences of the above factors on motor iron loss need to be considered.

The losses of silicon steel sheets are affected by temperature, which decrease with temperature increasing. Andreas Krings and Oskar Wallmark studied the effects of motor operating temperatures on iron losses and magnetic properties [13]. The iron loss models which consider temperature effects are proposed based on a large number of experiments [14]. Some researchers have corrected the temperature coefficient through experiment measurements, while the correctness of the models is verified by silicon steel sheets and motor [15], [16]. Due to the difficulty in heat dissipation for HSPMSMs, over high temperature problems need to be avoided for motor during operation. Therefore, it is necessary to consider the influences of temperature in the iron loss calculation for HSPMSMs.

The loss characteristics of the silicon steel sheet vary with stress. Studies indicate that the loss of silicon steel sheets increases with compressive stress, while decreases with tensile stress [17]. When the compressive stress is increased from 0 
MPa to $100 \mathrm{MPa}$, the unit loss of a certain type of silicon steel sheet doubles [18], [19]. The effect from stress on the silicon steel sheets' loss is limited. When the stress reaches to a certain value, the loss then maintains at a stable value [20]. The motor iron loss can be affected by both compressive stress and electromagnetic stress, which are generated during motor assembly process and operation [21]. The influences of stress also need to be included in motor iron loss calculation for HSPMSMs.

In this paper, the improved iron loss models considering different physical factors are proposed. Then, a HSPMSM, whose maximum speed up to $30,000 \mathrm{r} / \mathrm{min}$ is taken as an example to analyze the magnetic field, temperature field and stress field. Finally, the iron losses for the motor under different conditions are calculated by the proposed models, with their results compared to summary some conclusions. The accuracy of the proposed models is verified on the silicon steel sheet and the motor. The work done in this paper provides a valuable reference for the research of motor iron loss accurate calculation.

\section{IRON LOSS MODEL}

\section{A. The iron loss separation model}

The iron loss separation model was proposed by Bertotti, which can be divided into the hysteresis loss, eddy current loss and additional loss [22]. Its specific expression is:

$$
\begin{aligned}
p_{\mathrm{Fe}} & =p_{\mathrm{h}}+p_{\mathrm{c}}+p_{\mathrm{e}} \\
& =k_{\mathrm{h}} f B_{\mathrm{m}}^{\alpha}+\frac{d^{2}}{12 \delta \rho} \int_{0}^{T}\left|\frac{d B}{d t}\right|^{2} d t+k_{\mathrm{e}} f \int_{0}^{T}\left|\frac{d B}{d t}\right|^{1.5} d t
\end{aligned}
$$

Where, $p_{\mathrm{h}}$ is hysteresis loss. $p_{\mathrm{c}}$ is eddy current loss. $p_{\mathrm{e}}$ is additional loss. $k_{\mathrm{h}}$ and $\alpha$ are hysteretic loss coefficients. $k_{\mathrm{e}}$ is additional loss coefficient. $d$ is the thickness of silicon steel sheet. $\delta$ is density. $\rho$ is the equivalent resistivity.

When excitation is sinusoidal, (1) can be simplified as:

$$
\begin{aligned}
p_{\mathrm{Fe}} & =p_{\mathrm{h}}+p_{\mathrm{c}}+p_{\mathrm{e}} \\
& =k_{\mathrm{h}} f B_{\mathrm{m}}^{\alpha}+k_{\mathrm{c}} f^{2} B_{\mathrm{m}}^{2}+k_{\mathrm{e}} f^{1.5} B_{\mathrm{m}}^{1.5}
\end{aligned}
$$

The additional loss is a small percentage of the motor iron loss. Therefore, in order to simplify the calculation of the iron loss, the additional loss term is not considered in this paper.

\section{B. Iron Loss Model with Magnetic-Effect}

Calculating iron loss ideally, the flux density waveform is sinusoidal and the magnetization is alternating magnetization. In fact, due to the influence of stator slotting and rotor structure, the flux density waveform is the superposition of fundamental wave and harmonics. And the induced current in the three-phase winding is incompletely symmetrical, resulting in the rotating magnetization.

The flux density waveform can be decomposed into a fundamental wave and many harmonics. So the rotating magnetization can be equivalent by two mutually orthogonal alternating magnetizations in the calculation process [23]. Hence, the expression for the iron loss model with magnetic-effect is:

$$
\begin{aligned}
p_{\mathrm{Fe}} & =p_{\mathrm{h}}+p_{\mathrm{c}} \\
= & k_{\mathrm{h}} \sum_{k=1}^{N} k f\left(B_{\mathrm{mkmax}}^{\alpha}+B_{\mathrm{mkmin}}^{\alpha}\right) \\
& +k_{\mathrm{c}} \sum_{k=1}^{N} k^{2} f^{2}\left(B_{\mathrm{mkmax}}^{2}+B_{\mathrm{mkmin}}^{2}\right)
\end{aligned}
$$

Where, $B_{\mathrm{mkmax}}$ and $B_{\mathrm{mkmin}}$ are the magnitudes of the flux density represented by the long and short axes in the elliptical rotating magnetization trajectory.

The loss characteristics of silicon steel sheet can be affected by high frequency significantly. The skin effect results in uneven distribution of eddy current on the laminates. It can be represented by a frequency coefficient [24].

$$
k_{\mathrm{c}}(f)=\frac{3}{D \sqrt{f}} \cdot \frac{\sinh (D \sqrt{f})-\sin (D \sqrt{f})}{\cosh (D \sqrt{f})-\cos (D \sqrt{f})}
$$

Where, $D=d \sqrt{\pi \mu \sigma} ; \mu$ is the average magnetic permeability of silicon steel sheet. $\sigma$ is the conductivity of silicon steel sheet.

\section{Iron Loss Model with Temperature-Effect}

The iron loss of silicon steel sheet decreases with temperature rising. The temperature effect on total loss can be reflected in hysteresis loss and eddy current loss respectively. $k_{\mathrm{h}}$ and $k_{\mathrm{c}}$ both vary with temperature. The original equation improved with temperature-effect is [14]:

$$
\begin{aligned}
p_{\mathrm{Fe}} & =p_{\mathrm{h}}+p_{\mathrm{c}} \\
& =k_{\mathrm{h}}(t) f B_{\mathrm{m}}^{\alpha}+k_{\mathrm{c}}(t) f^{2} B_{\mathrm{m}}^{2} \\
& =k_{\mathrm{h}}(t) f B_{\mathrm{m}}^{\alpha}+\frac{d^{2}}{12 \delta \rho(t)} f^{2} B_{\mathrm{m}}^{2}
\end{aligned}
$$

Due to the influence of equivalent resistivity by temperature, the temperature coefficient $k_{\mathrm{t}}$ is proposed for equivalent resistivity in the iron loss model. Assuming that equivalent resistivity of non-oriented silicon steel sheet increases linearly with temperature if $k_{\mathrm{t}}$ takes a positive value:

$$
\rho(t)=\rho_{0}\left(1+k_{\mathrm{t}} \cdot \Delta t\right)
$$

Eddy current coefficient is:

$$
k_{\mathrm{c}}(t)=\frac{d^{2}}{12 \delta \rho_{0}} \cdot \frac{1}{1+k_{\mathrm{t}} \cdot \Delta t}=k_{\mathrm{c} 0} \cdot \frac{1}{1+k_{\mathrm{t}} \cdot \Delta t}
$$

Where, $\rho_{0}$ is the base value of equivalent resistivity. $\Delta t$ is a temperature variation. $k_{\mathrm{t}}$ is the temperature coefficient, which is obtained by fitting iron loss curve under different temperature.

\section{Iron Loss Model with Stress-Effect}

Assuming that the direction of compressive stress acting on the silicon steel sheet is positive. Iron loss of silicon steel sheet increases with compressive stress. The microstructure of the silicon steel sheet is changed by compressive stress. The internal magnetic domain is newly arranged consuming more energy, which leads to loss increasing [17]. Therefore, the loss coefficient as a function of compressive stress is proposed. The iron loss model with stress-effect can be expressed as:

$$
\begin{aligned}
p_{\mathrm{Fe}} & =p_{\mathrm{h}}+p_{\mathrm{c}} \\
& =k_{\mathrm{h}}(\sigma) f B_{\mathrm{m}}^{\alpha}+k_{\mathrm{c}}(\sigma) f^{2} B_{\mathrm{m}}^{2}
\end{aligned}
$$


Where, $k_{\mathrm{h}}(\sigma)$ and $k_{\mathrm{c}}(\sigma)$ are the loss coefficient affected by the stress, which is obtained by fitting iron loss curve of stress using least mean square method.

\section{E. Iron Loss Model with Multi-physics Factors}

To analyze the relationship between each physical factor and the loss coefficient, the correlation coefficient is applied. Let (X, $\mathrm{Y})$ be a binary random variable, the correlation coefficient is defined as (9). The closer the correlation coefficient is to 1 , the higher the correlation between $\mathrm{X}$ and $\mathrm{Y}$.

$$
R=\frac{\operatorname{Cov}(X, Y)}{\sqrt{D X} \sqrt{D Y}}
$$

The correlation analysis between physical factors and loss coefficient is shown in Fig. 1. The correlation coefficient between stress and hysteresis loss coefficient is close to 1 . The correlation coefficient between temperature and eddy current loss coefficient is close to 1 . Therefore, the stress is the main factor affecting the hysteresis loss coefficient under different frequencies. And the temperature is the main factor affecting the eddy current loss coefficient under different frequencies.

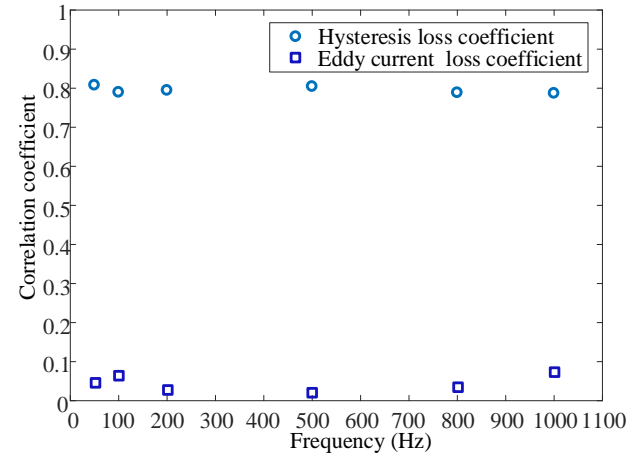

(a) Correlation between loss coefficients and stress under different frequencies

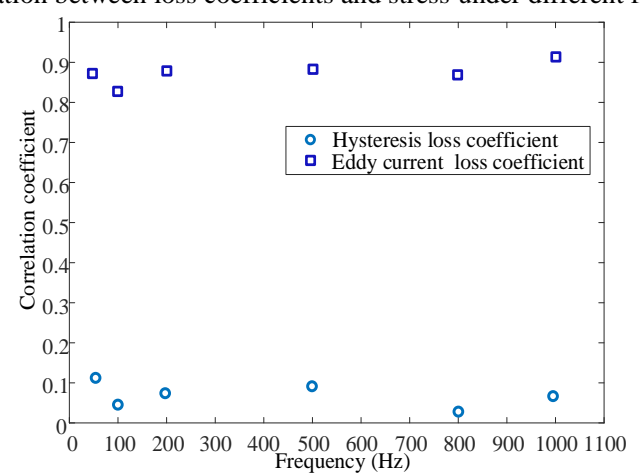

(b) Correlation between loss coefficients and temperature under different frequencies

Fig. 1 Correlation analysis of loss coefficient with stress and temperature

Based on the iron loss models considering different physical factors and the correlation analysis, the physical factors with the highest correlation are considered in the hysteresis loss and eddy current loss respectively. An iron loss model with multi-physics factors is proposed, as shown in (10).

$$
\begin{aligned}
p_{\mathrm{Fe}} & =p_{\mathrm{h}}+p_{\mathrm{c}} \\
= & k_{\mathrm{h}}(\sigma) \sum_{k=1}^{N} k f\left(B_{\mathrm{mkmax}}^{\alpha}+B_{\mathrm{mkmin}}^{\alpha}\right) \\
& +k_{\mathrm{c} 0}(f) \cdot \frac{1}{1+k_{\mathrm{t}} \cdot \Delta t} \sum_{k=1}^{N} k^{2} f^{2}\left(B_{\mathrm{mkmax}}^{2}+B_{\mathrm{mkmin}}^{2}\right)
\end{aligned}
$$

Where, $k_{\mathrm{h}}(\sigma), k_{\mathrm{c} 0}(f)$, and $k_{\mathrm{t}}$ are all loss coefficients which is obtained by fitting iron loss curves.

\section{FEM ANALYSIS OF HSPMSM}

\section{A. Structure and Main Paraments of HSPMSM}

The structure of HSPMSM analyzed in this paper is shown in Fig. 2. It adopts a two-pole and twenty-four-slot structure.

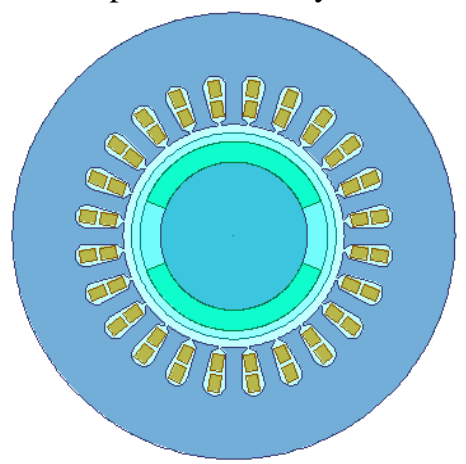

Fig. 2 The physical model of HSPMSM

The windings are connected in a double-layer short-distance distributed form as shown in Fig. 3. Three-phase windings A, B and $\mathrm{C}$ differ by $120^{\circ}$ electric angle in turn.

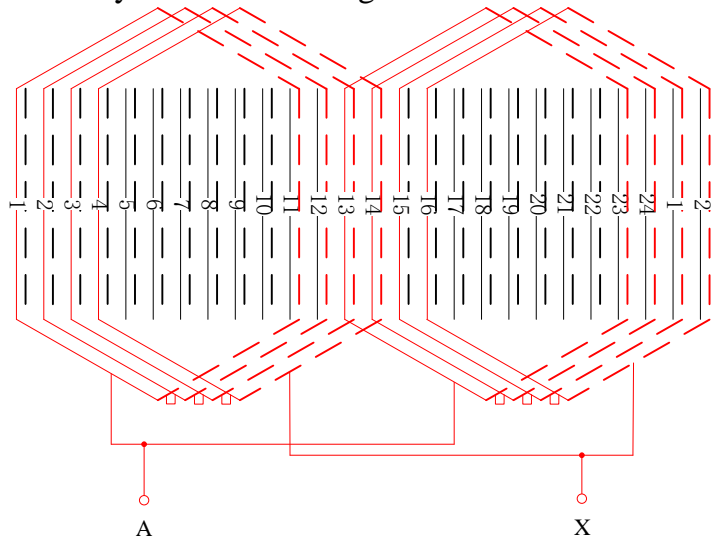

Fig. 3 Winding connection diagram of phase A

The rated parameters of HSPMSM are shown in Table I. TABLE I

THE MAIN RATED PARAMETERS OF HSPMSM

\begin{tabular}{cccc}
\hline \hline parameter & value & parameter & value \\
\hline Rated power / kW & 150 & $\begin{array}{c}\text { Stator outer/inner } \\
\text { diameter/mm }\end{array}$ & $250 / 125$ \\
Rated voltage / V & 380 & $\begin{array}{c}\text { Rotor outer diameter/ mm } \\
\text { Permanent magnet } \\
\text { thickness/mm }\end{array}$ & 108 \\
Rated speed / r/min & 30000 & 11.5 \\
\hline \hline
\end{tabular}

\section{B. Magnetic Field Analysis}

Due to the same trend of radial flux density waveform and the tangential one at the same position of the integer pitch of motor stator pitch, the feature points are taken only on one stator pitch. The distribution of feature points is shown in Fig. 4. The selection principle considering the top, middle and bottom of stator teeth and yoke are uniformly followed. 


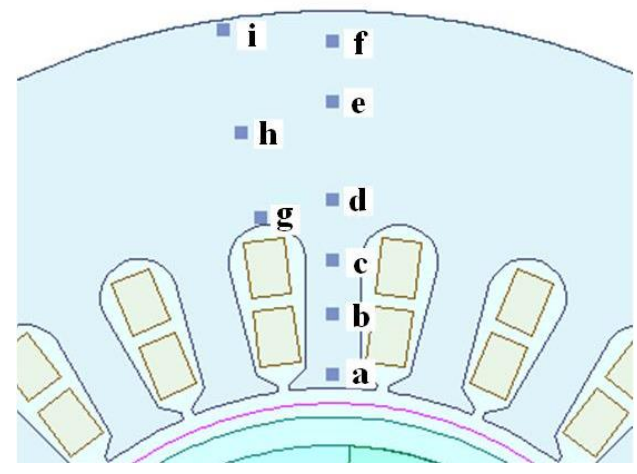

Fig. 4 The distribution of feature points on the stator

There are mainly two magnetization types include alternating magnetization and rotating magnetization. Due to the limitation of the paper length, only two representative points, which are shown in Fig. 5, are selected to illustrate the complex magnetization characteristics of the stator.

The flux density of point $c$ varies in both radial and tangential directions, indicating that its magnetization mode is alternating magnetization and rotating magnetization. The flux density of point $i$ changes only in the radial direction, indicating that its magnetization mode is only alternating magnetization. Hence, the alternating and rotating magnetization exist simultaneously in the stator.

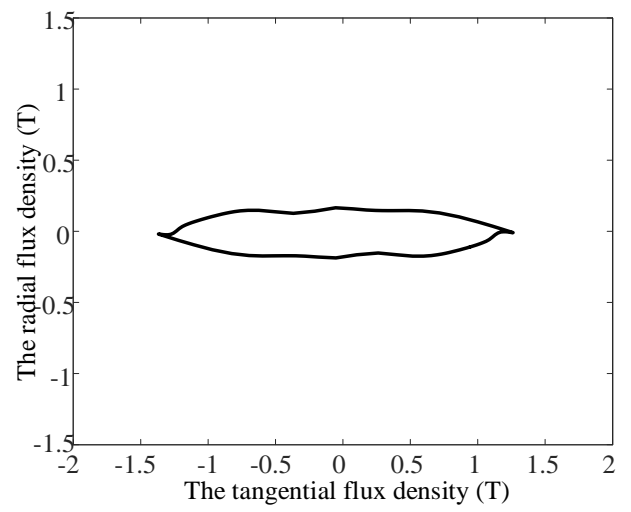

(a) feature point $\mathrm{c}$

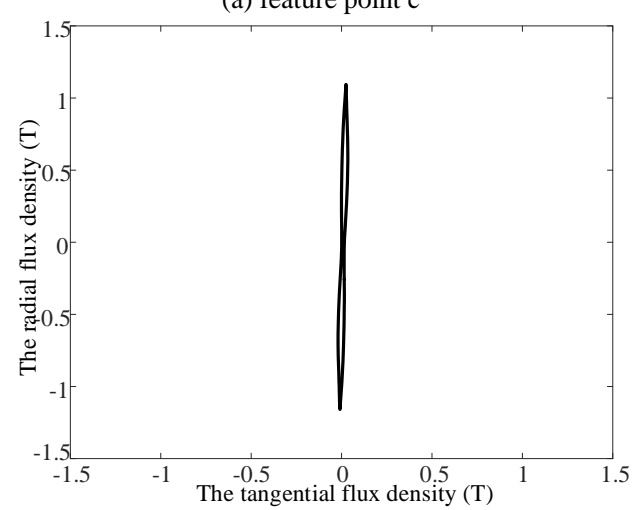

(b) feature point $\mathrm{i}$

Fig. 5 Magnetic tight track of feature points

Because of the existence of harmonics, the waveform of magnetization trajectory is distorted. The harmonic decomposed graphs of the feature points' magnetization track are shown in Fig. 6.

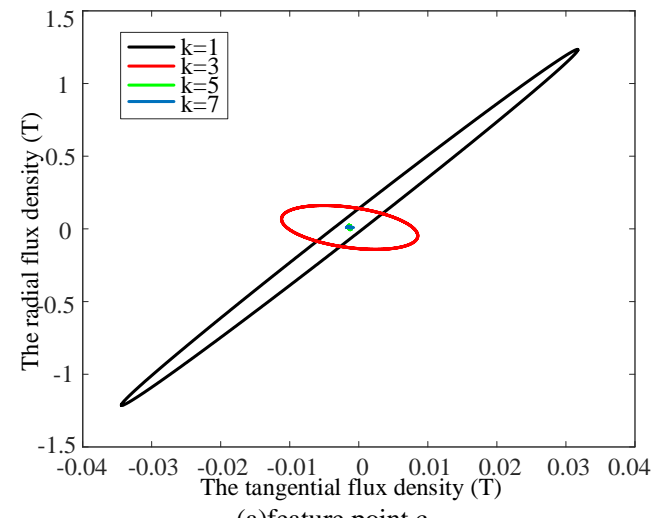

(a)feature point $\mathrm{c}$

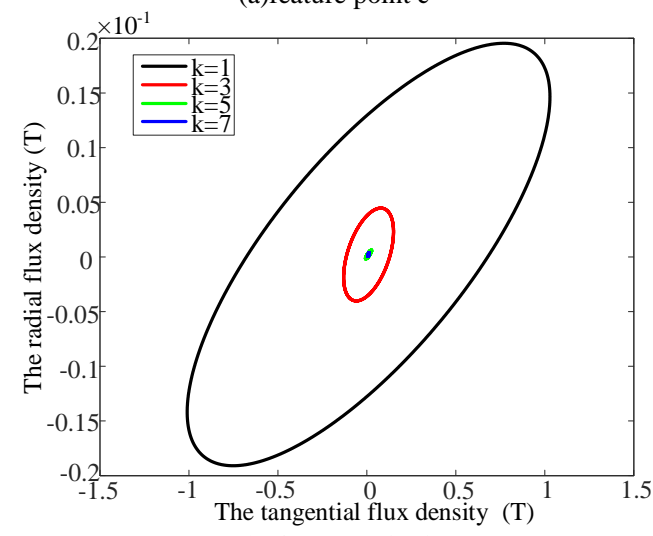

(b) feature point $\mathrm{i}$

Fig. 6 The harmonics decomposed graph of magnetization track

The harmonic content of each feature point is different. However, the third harmonic is the main harmonic component of all feature points, whose content can reach $48 \%$ of fundamental wave content. Hence, harmonic effects can not be ignored in the iron loss calculation.

\section{Temperature Field Analysis}

Assuming that there is no heat exchange between the motor stator and rotor, temperature rise calculation can be carried out by the two-heat-source heat circuit method [25]. The temperature distribution on motor stator can be observed by FEM method directly.

Under the natural heat dissipation condition, the boundary condition is set as:

(1) All circumferential symmetrical surfaces on both sides of the solution domain model are set as wall boundary conditions, and the heat transfer option is set as adiabatic.

(2) The contact surface between air gap and sheath is set as the boundary condition of rotating wall, and the angular velocity is set to $3140 \mathrm{rad} / \mathrm{s}(30000 \mathrm{r} / \mathrm{min})$ to simulate the actual rotation of the rotor.

The internal temperature field of motor is simulated and analyzed. The temperature distribution on the stator is shown in Fig. 7. 


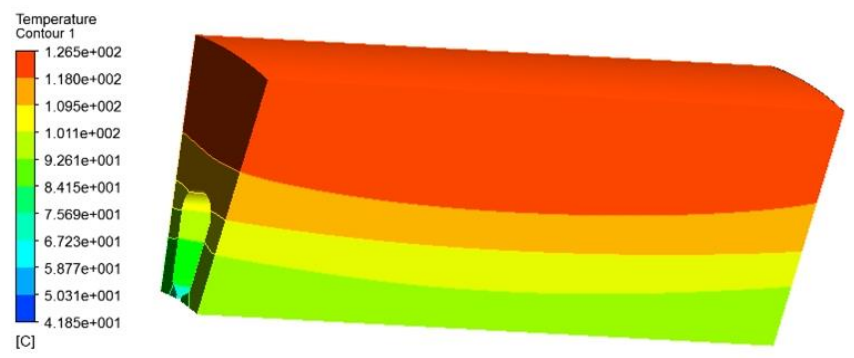

Fig. 7 Temperature field distribution of stator

It can be seen from the Fig. 7 that the stator temperature distribution of HSPMSM. And the maximum temperature can reach $126.5^{\circ} \mathrm{C}$. Hence, temperature effects can not be ignored in the iron loss calculation.

\section{Stress Analysis}

The stator is mainly subjected to mechanical compressive stress of the frame during assembly. The static mechanical compressive stress between the stator core and the frame is determined by the static interference [26]. According to the theory of thick-walled cylinders of elastic mechanics, the equation for calculating the static interference is [27]:

$$
\delta_{\mathrm{s}}=r_{\mathrm{so}}-r_{\mathrm{fi}}
$$

The interference between the stator and the frame is set as $0.19 \mathrm{~mm}$. The FEM simulation result is shown in Fig. 8 .
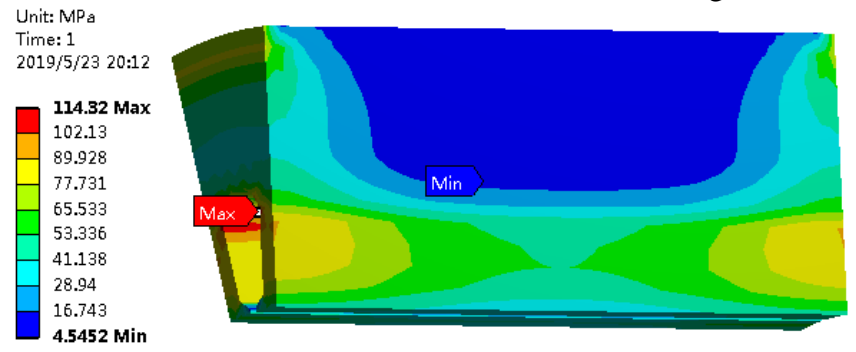

Fig. 8 Stress field distribution of stator

The maximum value occurs at the bottom of the stator slot which is $114.32 \mathrm{MPa}$. The minimum value occurs in the middle of the stator yoke which is $4.5452 \mathrm{MPa}$.

\section{IRON LOSS CALCULATION AND ANALYSIS}

\section{A. Measurement System}

In order to measure the magnetic properties of silicon steel sheets under different conditions, the monolithic measurement method is adopted. The schematic diagram and physical map are shown in Fig. 9.

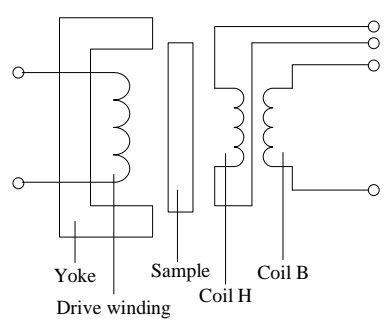

(a) Schematic diagram

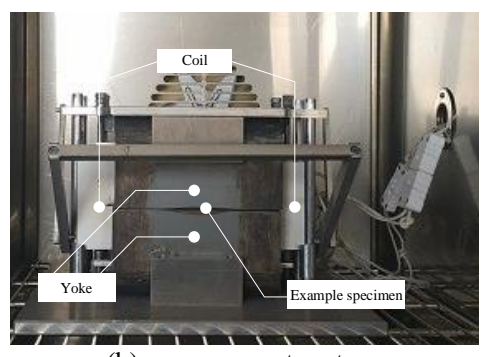

(b) measurement system

Fig. 9 Magnetic property test equipment for silicon steel sheet
Variables for measuring conditions include frequency, temperature, and stress. The measured frequency range is $50 \mathrm{~Hz}$ - $1 \mathrm{kHz}$. The measured temperature range is $20^{\circ} \mathrm{C}-200^{\circ} \mathrm{C}$. The measured stress range is $0 \mathrm{MPa}-100 \mathrm{MPa}$. And the example specimen of silicon steel sheet is B20AT1500.

\section{B. Calculation and Analysis of Loss Coefficient}

Based on the test data of the silicon steel sheets loss, the loss coefficients are fitted by a combination method of the Levenberg-Marquardt and the general global optimization method. The loss coefficients obtained by fitting at different frequencies $\left(\mathrm{T}=20^{\circ} \mathrm{C}, \sigma=0 \mathrm{MPa}\right)$ are shown in Table II. It can be seen that the hysteresis loss coefficient fluctuates in a small range and the eddy current loss decreases with the increase of frequency.

TABLE II

LOSS COEFFICIENTS

\begin{tabular}{ccc}
\hline \hline$f / \mathrm{Hz}$ & $k_{\mathrm{h}}$ & $k_{\mathrm{c}}$ \\
\hline 50 & 0.0336824 & 0.00024184 \\
200 & 0.0354872 & 0.00006541 \\
500 & 0.0344352 & 0.00004033 \\
800 & 0.0378121 & 0.00003176 \\
\hline \hline
\end{tabular}

The loss coefficients obtained by fitting at different temperatures $(f=500 \mathrm{~Hz}, \sigma=0 \mathrm{MPa})$ are shown in Table III. The hysteresis loss coefficient fluctuates in a small range and the temperature coefficient decreases with the increase of temperature. Due to the frequency remains the same, $k_{\mathrm{c} 0}$ is constant.

TABLE III

LOSS COEFFICIENTS

\begin{tabular}{ccc}
\hline \hline $\mathrm{T} /{ }^{\circ} \mathrm{C}$ & $k_{\mathrm{h}}$ & $k_{\mathrm{t}}$ \\
\hline 20 & 0.0344352 & 0.23970 \\
80 & 0.0317765 & 0.05176 \\
150 & 0.0336794 & 0.03814 \\
200 & 0.0324109 & 0.03110 \\
\hline \hline
\end{tabular}

According to the measured loss curves from $0 \mathrm{MPa}$ to $100 \mathrm{MPa}\left(f=500 \mathrm{~Hz}, \mathrm{~T}=20^{\circ} \mathrm{C}\right)$, the loss coefficients with the compressive stress is obtained in Table IV. The eddy current loss coefficient hardly varies with compressive stress (Table III), which indicates the independence between eddy current loss and stress. The hysteresis loss coefficient increases with compressive stress less than $40 \mathrm{MPa}$. With the compressive stress more than $40 \mathrm{MPa}$, the hysteresis loss coefficient starts to increase slowly. indicating that the hysteresis loss coefficient is affected by the compressive stress weakly.

TABLE IV

LOSS COEFFICIENTS

\begin{tabular}{ccc}
\hline \hline$\sigma / \mathrm{MPa}$ & $k_{\mathrm{h}}$ & $k_{\mathrm{c}}$ \\
\hline 0 & 0.0344352 & 0.0002403 \\
20 & 0.0422723 & 0.0002373 \\
40 & 0.0505116 & 0.0002395 \\
60 & 0.0525265 & 0.0002386 \\
80 & 0.0527531 & 0.0002391 \\
100 & 0.0529014 & 0.0002392 \\
\hline \hline
\end{tabular}




\section{Calculation and Analysis of Silicon Steel Sheets Loss}

In order to verify the accuracy of the iron loss model, the calculated and measured values of the silicon steel sheet loss are first compared. The average absolute error is used as one of the criteria for measuring the accuracy of the iron loss model.

The average absolute error is defined as:

$$
e_{\mathrm{r}}=\frac{1}{M} \sum_{i=1}^{M}\left(P_{\text {cal } i}-P_{\text {meai }}\right)
$$

Where, $P_{\text {cal } i}$ and $P_{\text {meai }}$ are the calculated and measured values of the loss, respectively. $M$ denotes the total counts of measurement.

The losses of silicon steel sheet from $50 \mathrm{~Hz}$ to $1 \mathrm{kHz}$ are calculated by the iron loss model considering high frequency, as shown in Fig. 10. The error between the calculated value and the measured one is less than $3.6 \%$.

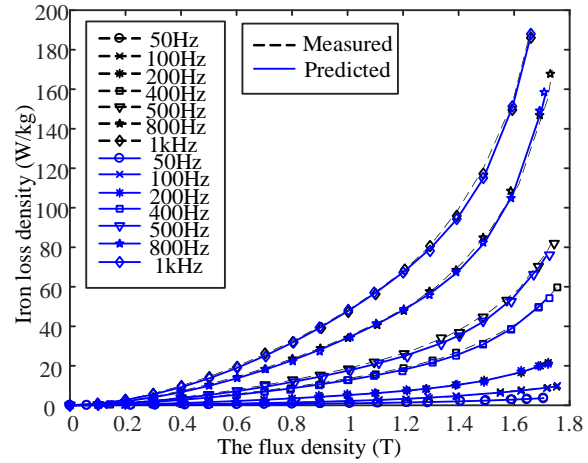

Fig. 10 Iron loss curves of various frequency

The measured iron loss and predicted one are exhibited in Fig. 11, respectively. Since the loss curves between different temperatures are relatively close, the two temperatures $\left(20^{\circ} \mathrm{C}\right.$ and $150^{\circ} \mathrm{C}$ ) are selected for illustration. The error between the calculated value and the measured one is less than $2.7 \%$ in the range from $20^{\circ} \mathrm{C}$ to $200^{\circ} \mathrm{C}$.

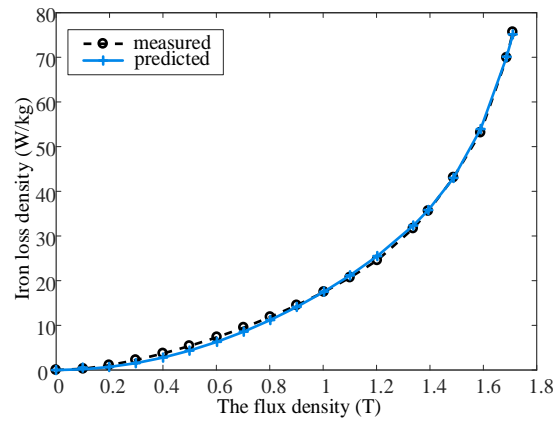

(a) $20^{\circ} \mathrm{C}$

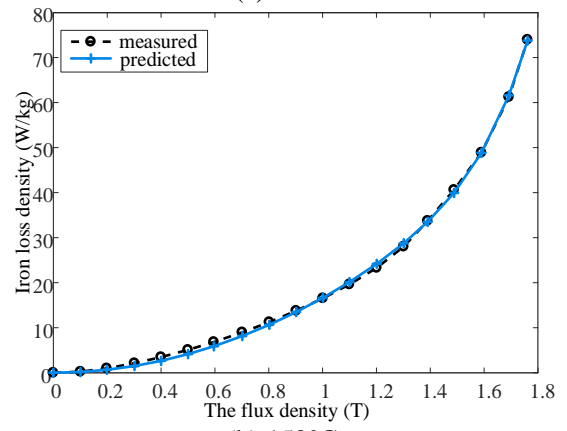

(b) $150^{\circ} \mathrm{C}$

Fig. 11 Iron loss curves of various temperature

The comparison between the measured iron loss and predicted values is shown in Fig 12, respectively. The iron loss gradually increases with the increase of the stress, but the growth rate is obviously slowed down from $60 \mathrm{MPa}$ to $100 \mathrm{MPa}$. The error between the calculated value and the measured one is less than $4.1 \%$ in the range from $0 \mathrm{MPa}$ to $100 \mathrm{MPa}$.

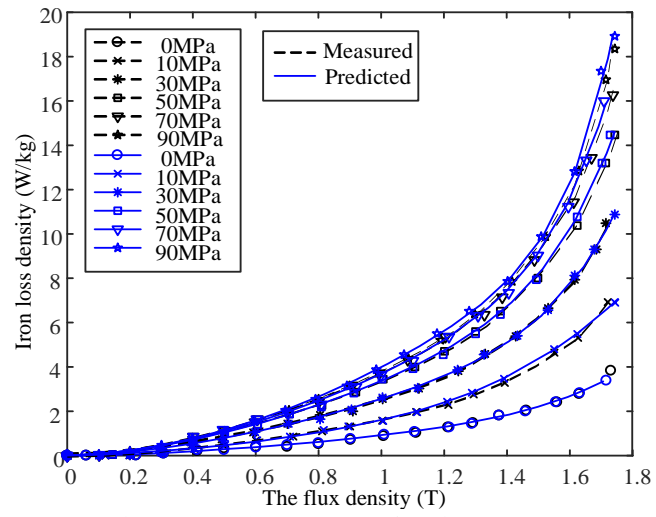

Fig. 12 Iron loss curves of various stress

\section{Comparison and Analysis of Iron Loss in HSPMSM}

In order to study the influence of single variable on iron loss of HSPMSM, the motor iron loss considering single physical factor is calculated. Applying the iron loss model with magnetic-effect, temperature-effect and stress-effect respectively, the motor iron loss is calculated. The motor iron loss increases with the increase of frequency as shown in Fig. 13. The motor iron loss decreases with the increase of temperature as shown in Fig.14. The motor iron loss increases first and then remains stable with the increase of stress as shown in Fig. 15.

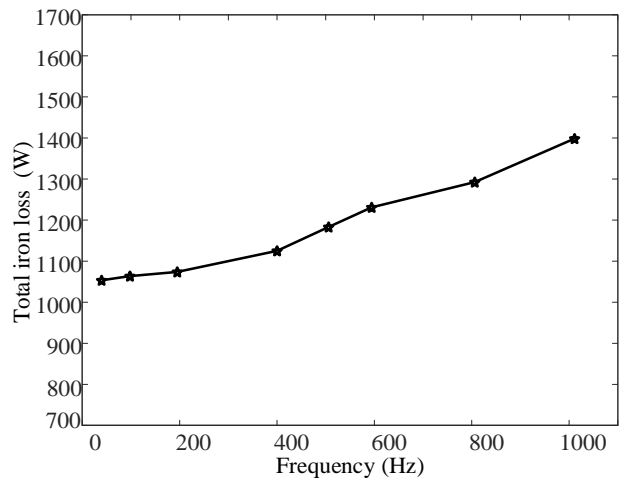

Fig. 13 Motor iron loss of various frequency

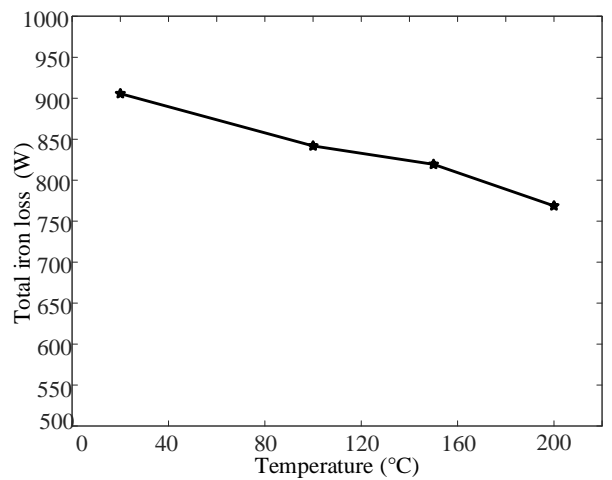

Fig. 14 Motor iron loss of various temperature 


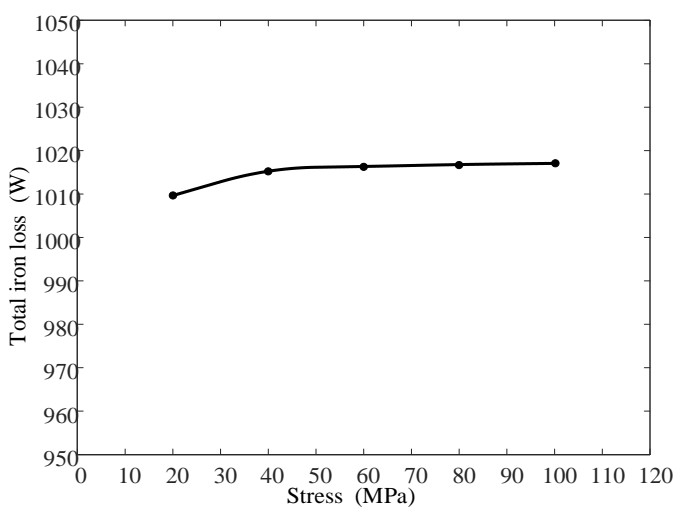

Fig. 15 Motor iron loss of various stress

Based on the FEM analysis of HSPMSM, the average temperature on the stator is about $84^{\circ} \mathrm{C}$ and the average stress is about $60 \mathrm{MPa}$ under no-load condition. The results of motor iron loss with the models considering different factors are shown in Table V.

TABLE V

IRON LOSS UNDER DIFFERENT CALCULATION MODELS

\begin{tabular}{cc}
\hline \hline Model & Value/W \\
\hline The iron loss separation model & 957.1 \\
Iron loss model with magnetic-effect & 1210.9824 \\
Iron loss model with temperature-effect & 841.8360 \\
Iron loss model with stress-effect & 1016.8244 \\
Iron loss model with multi-physics factors & 1291.8696 \\
\hline
\end{tabular}

The percentage variation of iron loss is used to express the effect for three factors on motor iron loss. The rotating magnetization, frequency, and harmonics of magnetic field, and the stress all result in the iron loss increasing, while the temperature result in the one decreasing. The higher percentage variation of iron loss, as shown in Fig.16, indicates that magnetic field is the mainly influencing factor of iron loss. And the effect of temperature field and stress field should not be ignored in motor iron calculation. The purpose of the above analysis is to figure out the influencing factors of iron loss, and analyze the severity of the impact of each factor, which is helpful to take corresponding measures to reduce iron loss.

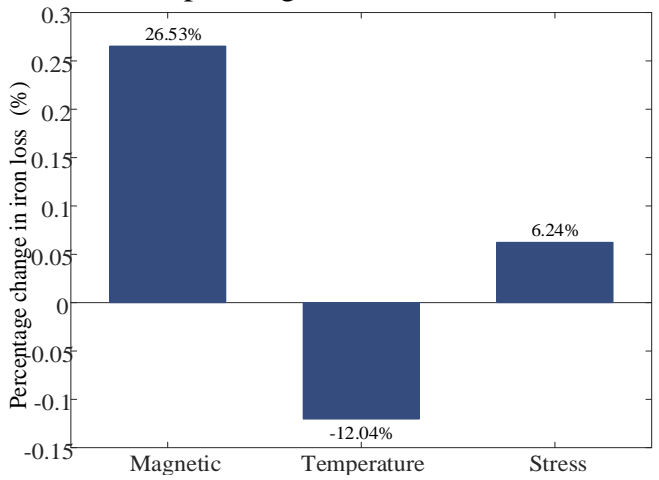

Fig. 16 The degree of influence of different physical factors on iron loss

In order to verify the accuracy of iron loss model considering multi-physics factors, the HSPMSM prototype shown in Fig. 17 is took as the test object. The iron loss of no-load motor can be obtained by the loss separation method. The measured iron losses at different voltages are compared with the calculated iron losses as shown in Fig. 18.

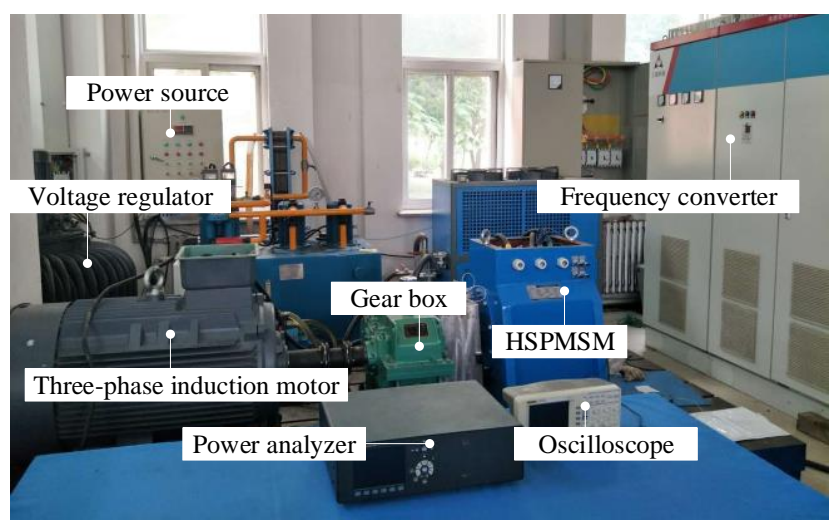

Fig. 17 Prototype measurement system

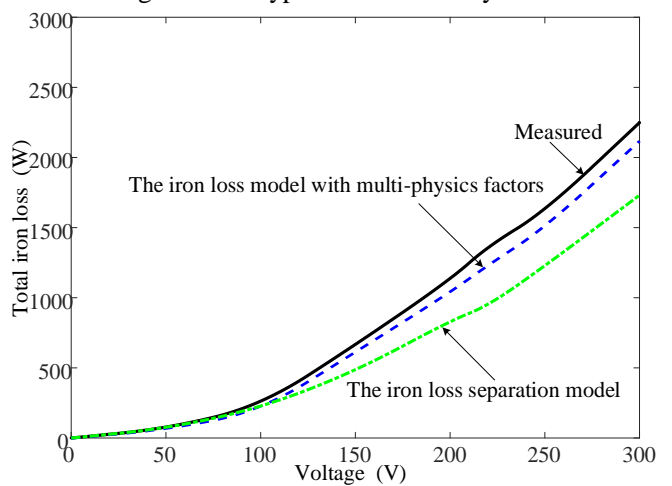

Fig. 18 Comparisons between calculated and measured iron losses

Based on the experimental results, it can be learnt that the calculated iron losses for the model with multi-physics factors are closer to the measured iron losses than the result of the iron loss separation model.

\section{CONCLUSION}

This paper analyzes the magnetic field, temperature field and stress field distribution characteristics of HSPMSM. The improved models of HSPMSM iron loss considering multi-physics factors are proposed. The following conclusions are obtained:

(1) The iron losses calculated by the model considering multi-physics factors are closer to the measured iron loss of prototype.

(2) The loss coefficients are affected by the frequency, temperature and stress. The hysteresis loss coefficient is mainly affected by stress, and the eddy current loss coefficient is mainly affected by frequency and temperature.

(3) The iron loss models considering different physical factors are validated by silicon steel sheet. The errors between calculated and measured values are less than $5 \%$ in all three cases.

(4) In HSPMSM, the calculated values of the iron loss model considering multi-physical factors are closer to the measured values, which proves that the accuracy of the model is higher than that of the iron loss separation model.

\section{REFERENCES}

[1] F. G. Zhang, G. H. Du, and T. Y. Wang, "Electromagnetic design and loss calculations of a 1.12-MW high-speed permanent magnet motor for compressor applications," IEEE Trans. Energy Convers., vol. 31, no. 1, pp. 132-140, Mar. 2016. 
[2] Y. Zhang, S. McLoone, and W. P. Cao, "Power loss and thermal analysis of a MW high speed permanent magnet synchronous machine," IEEE Trans. Energy Convers., vol. 32, no. 4, pp. 1468-1478, Dec. 2017.

[3] H. J. Wang, Z. Qu, and S. F. Tang, " Analysis and optimization of hybrid excitation permanent magnet synchronous generator for stand-alone power system," J. Magn. Magn. Mater., vol. 436, pp. 117-125, Aug. 2017.

[4] F. G. Zhang, R. Dai, G. W. Liu, and T. H. Cui, "Design of HSIPMM based on multi-physics fields," IET Electr. Power Appl., vol. 12, no. 8, pp. 1098-1103, Sept. 2017.

[5] J. X. Shen, P. Li, and H. Hao, "Study on electromagnetic losses in high-speed permanent magnet brushless machines-the state of the art," Proceedings of the CSEE, vol. 33, no. 3, pp. 62-74, Jan. 25, 2013.

[6] Y. Zhang, S. McLoone, and W. P. Cao. "High speed permanent magnet motor design and power loss analysis," ITEC Asia-Pacific 2017, Oct. 23, 2017

[7] N. Takahashi, Y. Mori, and Y. Yunoki, "Development of the 2-D single-sheet tester using diagonal exciting coil and the measurement of magnetic properties of grain-oriented electrical steel sheet," IEEE Trans. Magn., vol. 47, no. 10, pp. 4348-4351, Apr. 2011.

[8] H. Mikami, K. Ide, and S. Kikuchi, "Magnetic field analysis of a squirrel-cage induction motor considering rotor skew and higher harmonics in the secondary current," Electrical Engineering in Japan, vol. 129 , no. 2 , pp. $98-109$, Nov. 15, 2015.

[9] T. D. Strous, X. Z. Wang, and H. Polinder, "Brushless doubly fed induction machines: magnetic field analysis," IEEE Trans. Magn., vol. 52, no. 11, Nov. 2016.

[10] J. H. Seo, T. K. Chung, and C. G. Lee, "Harmonic iron loss analysis of electrical machines for high-speed operation considering driving condition," IEEE Trans. Magn., vol. 45, no. 10, pp. 4656-4659, Oct. 2009.

[11] D. Gerada, A Mebarki, and N. L. Brown, "High-speed electrical machines: technologies, trends, and developments," IEEE Trans. Ind. Electron., vol. 61, no. 6, pp. 2946-2959, June. 2014.

[12] S. L. Jiang, J. B. Zou, and Y. X. Xu, "Variable coefficient iron loss calculating model considering rotational flux and skin effect," Proceedings of the CSEE, vol. 31, no. 3, pp. 104-110, Jan. 25, 2011.

[13] A. Krings, S. A. Mousavi, and O. Wallmark, "Temperature influence of $\mathrm{NiFe}$ steel laminations on the characteristics of small slotless permanent magnet machines," IEEE Trans. Magn., vol.49, no.7, pp.4064-4067, 2013.

[14] J. Q. Chen, D. Wang, and S. W. Cheng, "Modeling of temperature effects on magnetic property of non-oriented silicon steel lamination," IEEE Trans. Magn., vol.51, no.11, Nov. 1, 2015.

[15] S. S. Xue, J. H. Feng, and S. Y. Guo, "A new iron loss model for temperature dependencies of hysteresis and eddy current losses in electrical machines," IEEE Trans. Magn., vol. 54, no.1, Jan. 2018.

[16] S. S. Xue, J. H. Feng, and S. Y. Guo, "Iron loss model under DC bias flux density considering temperature influence," IEEE Trans. Magn., vol. 53, no.11, Nov. 2017.

[17] D. Miyagi, Y. Aoki, and M. Nakano, "Effect of compressive stress in thickness direction on iron losses of non-oriented electrical steel sheet," IEEE Trans. Magn., vol.46, no.6, pp.2040-2043, June. 2010.

[18] Y. Oda, H. Toda, and N. Shiga, "Effect of Si content on iron loss of electrical steel sheet under compressive stress," IEEE Trans. Magn., vol.50, no.4, Apr. 1, 2014.

[19] Y. Oda, T. Hiratani, and S. Kasai, "Effect of compressive stress on iron loss of gradient Si steel sheet," IEEJ Trans. Ind. Appl., vol.135, no.12, pp.1199-1206, Dec. 1, 2015.

[20] M. Lobue, C. Sasso, and V. Basso, "Power losses and magnetization process in $\mathrm{Fe}-\mathrm{Si}$ non-oriented steels under tensile and compressive stress," J. Magn. Magn. Mater., vol.215, no.3, pp.124-126, June. 2, 2000.

[21] K. Fujisaki, R. Hirayama, and T. Kawachi, "Motor core iron loss analysis evaluating shrink fitting and stamping by finite-element method," IEEE Trans. Magn., vol.43, no.5, pp.1950-1954, May. 2007.

[22] Y. K. Huang, J. N. Dong, J. G. Zhu, and Y. G. Guo, "Core loss modeling for permanent magnet motor based on flux variation locus and finite element method," IEEE Trans. Magn., vol. 48, no. 2, pp. 1023-1026, Feb. 2012.

[23] S. Gorji Ghalamestani, L. Vandevelde, and J. Dirckx, "Magnetostriction and the influence of higher harmonics in the magnetic field," IEEE Trans. Magn., vol. 48, no. 11, pp. 3981-3984, 2012.
[24] E. Dlala, and A. Arkkio, "A general model for investigating the effects of the frequency converter on the magnetic iron losses of a squirrel-cage induction motor," IEEE Trans. Magn., vol. 45, no. 9, pp. 3303-3315, Sept. 2009

[25] Z. Y. Huang, J. C. Fang, and X. Q. Liu, "Loss calculation and thermal analysis of rotors supported by active magnetic bearings for high-speed permanent-magnet electrical machines," IEEE Trans. Ind. Electron., vol. 63, no. 4, pp. 2027-2035, April. 1, 2016.

[26] G. W. Liu, G. H. Qiu, and F. G. Zhang, "Outer rotor mechanical and dynamic performance analysis for high-speed machine," ITEC Asia-Pacific 2016, pp. 509-513, July. 13, 2016.

[27] T. Y. Wang, F. Q. Wen, and F. G. Zhang, "Analysis of multi-field coupling strength for MW high-speed permanent magnet machine," Transactions of China Electrotechnical Society, vol. 33, no.19, pp.4508-4516, Oct. 10, 2018.

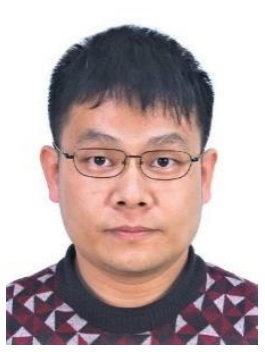

Guangwei Liu received the B.E.E., M.S.,and Ph.D. degrees from the Shenyang University of Technology, Shenyang, China, in 2005, 2008, and 2015, respectively, all in electrical engineering. He is currently an Associate Professor with the School of Electrical Engineering at Shenyang University of Technology, Shenyang, China. His major research interests and activities are in the area of PM electrical machines and its drive system.

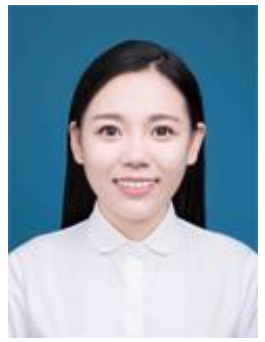

Meiyang Liu received her B.S. and M.S. degrees in Electrical Engineering from Shenyang University of Technology, Shenyang, China, in 2016 and 2019, respectively. She is currently working in Zhuhai Gree Electric Appliance Co., Ltd. Her main research interests include high-speed machine design and loss analysis.

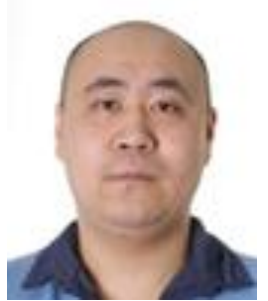

Yue Zhang was born in Shenyang, China. He received the B.Eng. degree from the Shenyang University of Technology, Shenyang, China, in 2011, the M.Eng. degree from Zhejiang University, Hangzhou, China in 2014, and the Ph.D. degree in Queen's University Belfast, Belfast, U.K., in 2018. He is now researching in University of Nottingham, UK. His research interests include the design and analysis of electrical machines for industrial applications and electrical vehicles.

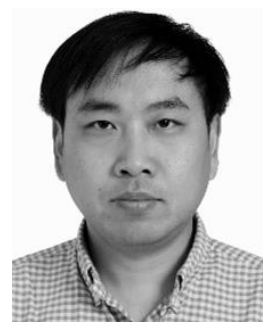

Huijun Wang received the M.S. degree in electrical engineering from Shenyang University of technology, Shenyang, China, in 2003 and Ph.D. degree in electrical and mechatronics engineering from Kyungsung University, Busan, Korea, in 2009. He is currently working in School of Instrumentation Science and Opto-electronics Engineering, Beihang University, Beijing, China. His research interest includes motor design and drive system.

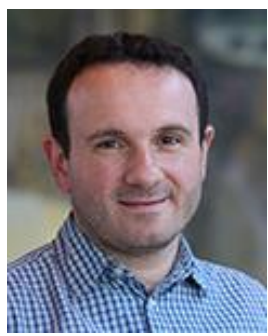

Chris Gerada received the Ph.D. degree in numerical modeling of electrical machines from The University of Nottingham, Nottingham, U.K., in 2005. He subsequently worked as a Researcher with The University of Nottingham on high-performance electrical drives and on the design and modeling of electromagnetic actuators for aerospace applications. His main research interests include the design and modeling of high-performance electric drives and machines 